\title{
MODELOS DE DEMANDA POR MANZANAS FRESCAS CHILENAS EN PAÍSES DE LA UNIÓN EUROPEA ${ }^{1}$
}

\author{
GERMÁN LOBOS ANDRADE²*, LORETO GUTIÉRREZ BRAVO ${ }^{3}$ Y KAREN PINTO BRISO ${ }^{3}$
}

\begin{abstract}
RESUMEN - Se usó la técnica de regresión de mínimos cuadrados ordinarios para estimar la demanda por importaciones de manzanas frescas exportadas por Chile en cinco países de la Unión Europea. Para las estimaciones se utilizaron observaciones anuales del periodo 1984 a 2003 . Los resultados demuestran que (1) la demanda por importaciones de manzanas frescas chilenas depende de las tradicionales variables ingreso o gasto en consumo per cápita, (2) los precios relativos y la variación del tipo de cambio real juegan un papel destacado en la explicación de la demanda por importaciones, y (3) la demanda responde de manera diferente frente a los cambios en las variables explicativas en cada mercado.
\end{abstract}

Términos para indexación: mínimos cuadrados ordinarios, demanda, precios relativos, tipo de cambio real.

\section{EQUAÇÕES DE DEMANDA PARA MAÇÃS FRESCAS, CHILENAS, EM PAÍSES DA UNIÃO EUROPÉIA}

RESUMO - Foi usada a técnica de regressão de mínimos quadrados ordinários para estimar a demanda das importações de maçãs frescas exportadas pelo cinco países da União Européia. Para fazer as estimações foram utilizadas observações anuais do período 1984 a 2003 . Os resultados demostram que (1) a demanda por importações de maçãs frescas chilenas depende das tradicionais variáveis ingresso ou gasto em consumo per cápita, (2) os preços relativos e a variação do tipo de câmbio real tem una importante participação na explicação da demanda por importações, e (3) a demanda responde de forma diferente com relação às mudanças nas variáveis explicativas em cada mercado.

Termos para indexação: mínimos quadrados ordinários, demanda, preços relativos, tipo de câmbio real

\section{INTRODUCCIÓN}

Según ODEPA (2004), durante el año 2003 las exportaciones de manzanas frescas chilenas alcanzaron 596,4 millones de kilogramos netos (KN), siendo los principales mercados de destino los países de América Latina (33,7\%), la Unión Europea (31,1\%) y EE.UU. (15,5\%). El mismo año, las principales variedades comercializadas en los mercados externos fueron Royal Gala (28,6\%), Granny Smith (19,2\%) y Richard Delicius $(15,3 \%)$; en menor medida se comercializaron Fuji $(7,8 \%)$, Red Starking $(6,7 \%)$, Braebum $(4,2 \%)$ y las demás variedades $(18,2 \%)$.

Hasta el año 2003, las manzanas frescas chilenas se comercializaban en todos los países que hasta esa fecha integraban la Unión Europea, excepto en Austria y Luxemburgo. Sin embargo, de acuerdo a las cifras de FAS/USDA (2004), históricamente los mercados más importantes, en términos de volumen, han sido Holanda, España, Reino Unido, Alemania e Italia. Los principales países competidores de las manzanas frescas chilenas en la Unión Europea son los otros países productores del Hemisferio Sur: Argentina, Australia, Brasil, Nueva Zelanda y Sudáfrica; además de EE.UU. y China.

La mayoría de los estudios de demanda por frutas (Richards, 2000; Lee, 1994) sugieren que las variables explicativas más frecuentemente utilizadas en las estimaciones son el ingreso per cápita (medido a través del producto interno bruto) y el precio. Sin embargo, Agbola (2003) y Stewart et al. (2003) incorporaron el gasto en consumo como variable explicativa de la demanda por frutas y vegetales. Duval et al. (2002), además de los tradicionales efectos, precio e ingreso, incluyeron el gasto en difusión de nuevos productos para estimar la demanda por frutas frescas tropicales en Indonesia, Malasia, Singapur, Francia y Reino Unido. En la estimación de modelos de demanda por importaciones de toronjas frescas, Lee y Fairchild (1988) consideraron como variables explicativas el tipo de cambio real, en unidades de moneda extranjera por moneda local, y el precio relativo de las toronjas frescas con respecto al precio de los otros bienes disponibles, además del gasto o ingreso total de todos los consumidores. El efecto del gasto en programas de promoción de exportaciones sobre la demanda por importaciones fue incluido por Kaiser et al. (2003).
El estudio desarrollado por Lee (1994), consideró un sistema diferencial de demanda para estimar elasticidades precio compensadas para una canasta de frutas frescas (manzanas, mandarinas, uvas, fresas, bananas y limones) en Japón, utilizando datos anuales del periodo 1973 a 1990. El autor concluyó que las manzanas son un bien normal, obteniendo una elasticidad ingreso de la demanda de 0,2194; además, la demanda por manzanas es inelástica al precio, observando una elasticidad precio compensada de $-0,7925$.

Agbola (2003), investigó los patrones de demanda agregada por alimentos en Sudáfrica utilizando datos de corte transversal del año 1993, mediante una aproximación lineal de un Sistema Casi Ideal de Demanda (LA/AIDS), de Deaton \& Muellbauer (1980), basado en el gasto en carnes y pescados, granos, productos lácteos, frutas, vegetales, y otros alimentos, para una muestra de 9.000 hogares. En el caso de las frutas, el autor estimó las elasticidades precio de la demanda Marshalliana $(-0,263)$ e Hicksiana $(-0,225)$, además de la elasticidad gasto $(0,717)$, la propensión media a consumir frutas $(5 \%)$ y la propensión marginal al consumo (3,6\%). Agbola (2003) concluyó que la demanda por frutas es inelástica al precio y al ingreso, sugiriendo que los cambios en el ingreso deberían provocar un pequeño impacto sobre el gasto en frutas.

Stewart et al. (2003) usaron datos de corte transversal de 5.179 hogares de Estados Unidos, los cuales fueron estratificados por nivel de ingreso; en base a curvas tradicionales de Engel, examinaron las relaciones entre el ingreso de los hogares y el gasto en frutas y vegetales. Los autores concluyeron que las familias pobres gastan menos en frutas y vegetales que las familias no pobres; sin embargo, las familias pobres no incrementan el gasto en frutas y vegetales en respuesta a cambios pequeños en el ingreso, mientras que, dada una transferencia de riqueza lo suficientemente alta como para dejar la línea de pobreza, se podría esperar un mayor gasto en frutas y vegetales; por lo tanto, para los hogares no pobres las frutas y vegetales pueden clasificarse como bienes normales.

En base a datos anuales del periodo 1972 a 1986, Lee \& Fairchild (1988) utilizaron el modelo de ecuaciones aparentemente no relacionadas (SUR), propuesto inicialmente por Zellner (1962), para analizar las relaciones entre el tipo de cambio y la demanda por importaciones en

\footnotetext{
${ }^{1}$ (Trabalho 130/2004). Recebido: 10/09/2004. Aceito para publicação: 06/04/2005. Esta publicación fue financiada por el Fondo de Desarrollo Académico de la Facultad de Ciencias Empresariales de la Universidad de Talca.

${ }^{2}$ Ing. Comercial, Magíster en Economía Agraria (Pontificia Universidad Católica de Chile), Profesor Asociado de la Universidad de Talca, Facultad de Ciencias Empresariales, Escuela de Ingeniería Comercial. Teléfono: (56 71) 200330. Casilla 721, Talca, Chile. E-mail: globos@ utalca.cl * Autor para correspondencia ${ }^{3}$ Ingeniero Comercial, Universidad de Talca, Chile.
} 
Canadá, Japón y Europa de toronjas frescas exportadas por Estados Unidos. Los autores estimaron las elasticidades precio relativo de la demanda de toronjas frescas, ajustado por el tipo de cambio real, con respecto al precio de los otros bienes disponibles, ajustados por la inflación doméstica, en Canadá (-0,2846), Japón (-0,4791) y Europa (1,014); además, para mostrar la importancia del tipo de cambio real, excluyeron esta variable y estimaron las elasticidades precio relativo de la demanda de toronjas frescas con respecto al precio de los otros bienes disponibles en Canadá (-0,4667), Japón (-0,5682) y Europa (-0,3595). La principal conclusión de esta investigación sugiere que, aunque la demanda por toronjas frescas responde de diferente manera a los cambios en el precio relativo en los mercados analizados, el tipo de cambio real juega un importante rol en la estimación de la demanda por importaciones.

En base a observaciones anuales del periodo 1973 a 1987, Lee et al. (1990), basados en ecuaciones individuales, estimaron modelos lineales de demanda por importaciones de Japón de cítricos frescos (limones, naranjas y toronjas), otras frutas frescas (bananas y piñas) y jugos concentrados de cítricos provenientes de diferentes países exportadores (Argentina, Brasil, Israel, Estados Unidos y el resto del mundo), con el objeto de explicar la posición competitiva de los cítricos exportados por Estados Unidos en el mercado japonés. Las elasticidades precio de la demanda por importaciones obtenidas fueron inelásticas al precio para limones $(-0,8273)$, naranjas $(-0,4032)$, bananas $(-0,558)$; y elásticas al precio para toronjas $(-1,428)$ y piñas $(-1,1421)$.

Para estimar la demanda por frutas frescas tropicales en Francia y Reino Unido, Duval et al. (2002) usaron cinco modelos diferentes: un modelo estándar de difusión (SDIF), un modelo de demanda doble-log (DLD), un modelo de demanda lineal de difusión (LDDIF), un modelo de demanda de difusión de Gompertz (GDDIF), propuesto originalmente por Dixon (1980), y un modelo de demanda dinámico de difusión (DDDIF). En todos los modelos, los resultados sugieren que la demanda por frutas frescas tropicales es inelástica al precio, excepto en el caso del modelo DLD; además, en todos los casos, la demanda es inelástica al ingreso (bienes normales), excepto en el modelo DLD para Francia, en que la demanda es elástica al ingreso (bienes superiores). La principal conclusión de los autores es que el proceso de difusión es significativo, lo cual sugiere que los efectos del precio e ingreso sobre el consumo pueden estar sobrestimados cuando se utilizan los modelos de demanda tradicional en las estimaciones.

Finalmente, Cerda et al. (2004), estimaron elasticidades de demanda por manzanas frescas chilenas en el mercado de la Unión Europea considerado como un todo, utilizando datos anuales del periodo 1984 a 2002. En un modelo doble-log de demanda por importaciones, los autores concluyeron que las variables explicativas del modelo fueron el producto interno bruto per cápita de la Unión Europea, el precio relativo de las manzanas chilenas con respecto al precio de las manzanas provenientes de China, el factor de variación del tipo de cambio promedio ponderado de los países de la Unión Europea, y una variable dicótoma de cambio puntual, la cual permitió capturar el brusco aumento de las importaciones de manzanas chilenas durante los años 1991 y 1992. Los resultados obtenidos por Cerda et al. (2004) indicaron que la demanda por manzanas chilenas en la Unión Europea es inelástica con respecto al ingreso $(0,913)$, al precio relativo $(-0,368)$ y al tipo de cambio $(-0,519)$.

El objetivo principal de este trabajo fue plantear modelos econométricos de demanda por importaciones de manzanas frescas chilenas en cinco países de la Unión Europea (Alemania, Bélgica, España, Francia y Holanda), y estimar las respectivas elasticidades. Se espera que lo anterior contribuya a un conocimiento más específico de cada uno de dichos mercados y a disminuir el sesgo de la agregación sobre países del estudio realizado por Cerda et al. (2004).

\section{MATERIALES Y MÉTODOS}

La información utilizada en las estimaciones fue obtenida de diversas fuentes: Oficina de Estudios y Políticas Agrarias (ODEPA, 2004), Banco Mundial (2004), EUROSTAT (2004), FAOSTAT (2004), Bureau of
Labour Statistics (BLS, 2004) y del Banco Central Europeo (ECB, 2004). Los datos corresponden a series de tiempo anuales del período 1984 a 2003 ( $n=20$ ). Utilizando la técnica de MCO, se plantearon modelos de demanda uniecuacionales por importaciones de manzanas frescas chilenas y se estimaron las respectivas elasticidades en cinco países de la Unión Europea: Alemania, Bélgica, España, Francia y Holanda.

\section{Variables y datos}

Las variables utilizadas para especificar los modelos de demanda fueron las siguientes:

$\mathrm{M}_{\mathrm{i}}$ : importaciones totales de manzanas frescas chilenas realizadas por el país i de la Unión Europea, expresadas en KN.

$\mathrm{PIBPC}_{\mathrm{i}}$ : Producto interno bruto per cápita del país i de la Unión Europea, expresado en dólares de 2003, como variable proxy del ingreso per cápita.

$\mathrm{GCPC}_{\mathrm{i}}$ : Gasto en consumo per cápita del país i de la Unión Europea, expresado en dólares de 2003, como variable proxy del ingreso per cápita.

PIBPPC: Producto interno bruto per cápita a "poder de paridad de compra" del país i de la Unión Europea, expresado en dólares de 2003, como variable proxy del ingreso per cápita.

TCR: Tipo de cambio real del país i de la Unión Europea, expresando en unidades de moneda local de cada país por dólar de EE.UU., en moneda de 2003.

VTCR : Variación del tipo de cambio real del país i de la Unión Europea, expresado en porcentaje.

$\mathrm{PCIF}_{\text {Chile,s,i: }}$ : Precio CIF real de las manzanas frescas chilenas en la país i de la Unión Europea, expresado en dólares de 2003 por KN.

PCIF $_{\mathrm{s}, \mathrm{i}}$ : Precio CIF real de las manzanas provenientes del país $\mathrm{s}$ competitivo de Chile, en el país i de la Unión Europea, expresado en dólares de 2003 por KN.

$\mathrm{PCIF}_{\text {Chile,s,i }}:$ Precio relativo CIF real de las manzanas frescas chilenas con respecto al precio de las manzanas provenientes del país $\mathrm{s}$ competitivo de Chile, en el país i de la Unión Europea, expresado en dólares de 2003 por KN.

$\mathrm{PCIF}_{\mathrm{s} 1, \mathrm{~s} 2, \mathrm{i}}:$ Precio relativo CIF real de las manzanas frescas del país sustituto $\mathrm{s}_{1}$ con respecto al precio de las manzanas provenientes del país $\mathrm{s}_{2}$ competitivo distinto de Chile, en el país i de la Unión Europea, expresado en dólares de 2003 por KN.

$D_{\text {: }}$ Variable dicotómica (binaria) de cambio estructural o puntual en el país i de la Unión Europea, que asume un valor igual a cero o 1.

Los datos de demanda por importaciones de manzanas frescas chilenas (en volumen físico) y el precio FOB nominal fueron obtenidos de ODEPA (2004). Los datos del PIBPC GCPC $_{i}$, PIBPPC $_{i}$ e Índice de Precios al Consumidor (IPC.) se obtuvieron del Banco Mundial (2004). Además, la variación del $\mathrm{PIB}_{\mathrm{i}}$ para el año 2003 fue obtenida de EUROSTAT (2004). El Índice de Precios al Consumidor Armonizado ( HICP $_{\mathrm{s}}$ ) de la Unión Europea se obtuvo del Banco Central Europeo (ECB, 2004). Por otra parte, las exportaciones totales de manzanas frescas (volumen y monto) de los países del Hemisferio Sur, EE.UU. y China, y el precio FOB nominal, fueron obtenidos de FAOSTAT (2004). Considerando que lo EE.UU. es uno de los países más importantes en la producción y comercialización de manzanas frescas a nivel mundial, como indicador de la inflación internacional se utilizó la variación del Índice de Precios de Importaciones y Exportaciones (IEPI) de EE.UU., reportado por la BLS (2004).

Se realizaron ajustes a todas las variables utilizadas en la estimación de los modelos de demanda. La variable ingreso, medida como PIBPC $_{i}$ o GCPC , y disponible en moneda de 1995, fue convertida a moneda de 2003 utilizando como deflactor el IPC $_{\mathrm{i}}$. La variable ingreso, medida como PIBPPC , y disponible en moneda de 1995, fue convertida a moneda de 2003 utilizando como deflactor el HICPs de la Unión Europea. Para el año 2003, el PIBPC $_{i}$ se obtuvo de acuerdo al crecimiento anual estimado por EUROSTAT (2003) y el GCPC fue estimado de acuerdo al porcentaje promedio del ingreso dedicado al consumo durante los años en estudio. 
El TCR fue estimado multiplicando el tipo de cambio nominal $\left(\mathrm{TCN}_{\mathrm{i}}\right)$ de cada año por el IPC $_{\mathrm{i}}$ y dividiéndolo por el correspondiente IEPI de EE.UU. (Ossa, 2001). De esta forma, el TCR fue estimado como (1):

$\mathrm{TCR}_{\mathrm{it}}=\frac{\mathrm{TCN}_{\mathrm{it}} * \mathrm{IPC}_{\mathrm{it}}}{\mathrm{IEPI}_{\mathrm{t}}}$

Donde el subíndice t es el año respectivo.

Los precios FOB nominales de las manzanas exportadas por los países del Hemisferio Sur, EE.UU. y China fueron convertidos a precios FOB reales, en moneda de 2003, utilizando como deflactor el IEPI de EE.UU. De esta forma, el PCIF Chile, $_{\text {y }}$ PCIF $_{\mathrm{s}, \mathrm{i}}$ fueron obtenidos ajustando los precios FOB reales por el costo de seguro y flete. Por último, el

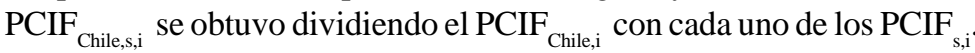
Este mismo procedimiento fue utilizado para calcular los precios relativos entre países sustitutos.

\section{Especificación de los modelos de demanda}

En todas las estimaciones de demanda se trabajó con modelos uniecuacionales, lo cual significa que la demanda por importaciones de manzanas frescas chilenas en cada país de la Unión Europea se determina de manera independiente. Según Gujarati (2000), los modelos uniecuacionales tienen una variable dependiente que es función de una o más variables explicativas, por lo que la relación "causa-efecto" que existe entre la variable dependiente y cada una de las variables explicativas es unidireccional, es decir, las variables explicativas son la "causa" y la variable dependiente es el "efecto". Bajo este supuesto, se pueden estimar los parámetros de una ecuación de demanda en forma aislada, sin considerar la información proporcionada por otras ecuaciones en el sistema de demanda.

Las demandas por manzanas frescas chilenas en Alemania, Bélgica, España, Francia y Holanda fueron estimadas utilizando tres diferentes modelos: modelo de demanda doble-log (DDL) como en (2), modelo de demanda semi-log (DSL) como en (3), y modelo de demanda lineal (MDL) como en (4).

DDL: $\ln \mathrm{M}_{\mathrm{i}}=a_{0}+a_{1} \ln \mathrm{I}_{\mathrm{i}}+\mathrm{a}_{2} \ln \mathrm{TC}_{\mathrm{i}}+\mathrm{a}_{3} \ln \mathrm{P}_{\mathrm{i}}+\mathrm{a}_{4} \mathrm{D}_{\mathrm{i}}+\mathrm{a}_{\mathrm{i}}$

DSL: $\ln \mathrm{M}_{\mathrm{i}}=\mathrm{a}_{0}+a_{1} \mathrm{I}_{\mathrm{i}}+\mathrm{a}_{2} \mathrm{TC}_{\mathrm{i}}+\mathrm{a}_{3} \mathrm{P}_{\mathrm{i}}+\mathrm{a}_{4} \mathrm{D}_{\mathrm{i}}+\mathrm{a}_{\mathrm{i}}$

MDL: $M_{i}=a_{0}+a_{1} I_{i}+a_{2} T_{i}+a_{3} P_{i}+a_{4} D_{i}+a_{i}$

Donde $\mathrm{I}_{\mathrm{i}}$ es la variable proxy del ingreso per cápita del país i de la Unión Europea, pudiendo estar representado por alguna de las siguientes variables: PIBPC $_{i}, \mathrm{GCPC}_{i}$ o PIBPPC $_{i} ; \mathrm{TC}_{i}$ es la variable proxy del tipo de cambio del país i de la Unión Europea, representado por una de las siguientes variables: $\mathrm{TCR}_{\mathrm{i}}$ o VTCR $\mathrm{V}_{\mathrm{i}}$ es la variable proxy del precio, representado por una o una combinación de las siguientes variables: $\mathrm{PCIF}_{\text {Chile, }}$ (precio-propio), $\mathrm{PCIF}_{\mathrm{s}, \mathrm{i}}$ (precio de sustituto), $\mathrm{PCIF}_{\text {Chile, }, \mathrm{i}}$ (precio relativo) y $\mathrm{PCIF}_{\mathrm{s} 1, \mathrm{~s} 2, \mathrm{i}}$ (precio relativo entre dos países competidores de Chile); $\mathrm{D}_{\mathrm{i}}$ es la variable dicotómica; y $\mathrm{a}_{\mathrm{i}}$ es el error aleatorio.

Las estimaciones fueron realizadas utilizando el software SPSS 11.5 .

\section{Pruebas estadísticas}

En cada modelo, para medir el porcentaje de variación total en la variable dependiente que es explicada por las variables explicativas, se usó el coeficiente de correlación múltiple $\mathrm{R}^{2}$ y $\mathrm{R}^{2}$ - ajustado. La global de cada modelo se midió con el estadístico de prueba F - Fisher. Para probar la significancia de cada variable explicativa se usó el estadístico $\mathrm{t}$ - student.

Se verificó el cumplimiento de lo existencia de los supuestos de multicolinealidad, heterocedasticidad y autocorrelación. Para detectar la presencia de multicolinealidad se utilizó la siguiente regla práctica: "si el $\mathrm{R}^{2}$ es alto, el estadístico $\mathrm{F}$ es significativo, pero cada $\mathrm{t}$ - calculado no es significativo, se tiene una probabilidad alta de que exista multicolinealidad"; el grado de multicolinealidad existente en los diversos modelos se verificó mediante la matriz de varianza-covarianza. Para la verificación del supuesto de no existencia de heterocedasticidad se utilizó la prueba de Sperman. Para verificar la no existencia de autocorrelación serial positiva o negativa se utilizó el estadístico d de Durbin-Watson. Finalmente, se utilizó el test de Shapiro-Wilks para no rechazar la hipótesis de que los residuos se distribuyen en forma normal.

\section{Signos esperados para los coeficientes}

Para cada modelo el signo del coeficiente de la variable $\mathrm{I}_{\mathrm{i}}$ puede ser positivo mayor que 0 y menor que 1 (bien normal), positivo mayor que 1 pero "bajo" (bien superior), positivo mayor que 1 pero "alto" (bien de lujo); cero (bien neutro); o negativo (bien inferior). La revisión de la literatura sugiere que las manzanas son un bien normal, lo cual significa que el signo esperado del coeficiente de la variable $I_{i}$ es positivo. Por lo tanto, frente a un cambio positivo en el ingreso, se esperaría que aumente la demanda por importaciones de manzanas frescas chilenas por parte del país i de la Unión Europea.

El signo del coeficiente de las variables $\mathrm{TCR}_{\mathrm{i}}$ o $\mathrm{VTCR}_{\mathrm{i}}$ en cada modelo se espera que sea menor que cero, es decir, ante un alza en cualquiera de ellas se espera que disminuya la demanda por importaciones de manzanas frescas chilenas por parte del país i de la Unión Europea, debido a que las manzanas frescas chilenas se vuelven relativamente más caras.

Para cada modelo el signo del coeficiente de las variables $\mathrm{PCIF}_{\text {Chile, } \mathrm{i}}$ y $\mathrm{PCIF}_{\text {Chile,s,i }}$ se espera que sea menor que cero, lo que indicaría que ante un aumento en el precio absoluto o relativo de las manzanas chilenas disminuya la demanda por importaciones de manzanas frescas chilenas en el país i de la Unión Europea. Sin embargo, para cada modelo, el signo del coeficiente de las variables PCIF $_{s, \mathrm{i}}$ y PCIF $_{\mathrm{s} 1, \mathrm{~s} 2, \mathrm{i}}$ se espera que sea mayor que cero, lo que implica que ante un aumento en el precio de las manzanas provenientes del país s del Hemisferio Sur, EE.UU. o China, competitivo de Chile, aumente la demanda por importaciones de manzanas chilenas en el país i de la Unión Europea (bienes sustitutos); en cambio para el segundo precio relativo puede que su signo sea también menor que cero, cuando la relación sea entre un país del Hemisferio Sur con uno del Hemisferio Norte. Lo que indica que ante un aumento en el precio de la manzanas del Hemisferio Norte, la demanda por manzanas chilenas podría aumentar.

Si la variable dicotómica $D_{i}$ es estadísticamente significativa, indicaría que existe un cambio estructural o puntual que estaría contribuyendo para explicar la variación de la demanda por importaciones de manzanas chilenas en el país i de la Unión Europea.

\section{Estimación de elasticidades}

Para el modelo DDL la elasticidad respectiva se obtuvo directamente del coeficiente á de cada variable explicativa j. En este caso, $c_{\mathrm{M}, \mathrm{ajj}}$ representa la elasticidad respectiva.

Para el modelo DSL la elasticidad respectiva se obtuvo como en (5).

$$
M, \quad \bar{X}_{j}
$$

Donde $\bar{X}_{j}$ representa el promedio simple de la variable respectiva $\mathrm{j}$ durante el período de estudio considerado.

En el caso del modelo MDL la elasticidad respectiva fue obtenida como en (6).

$$
{ }_{M, j} \quad j \frac{\bar{X}_{j}}{\bar{M}_{i}}
$$

Donde $\overline{\mathrm{X}}_{\mathrm{j}}$ representa el promedio simple de la variable respectiva j y $\overline{\mathrm{M}}_{\mathrm{i}}$ representa el promedio simple de las importaciones de manzanas frescas chilenas en el país i de la Unión Europea durante el período de estudio 
considerado.

\section{RESULTADOS Y DISCUSIÓN}

En la Tabla 1 se presentan las estadísticas de las variables utilizadas en las ecuaciones de demanda. Los valores presentados para los coeficientes (Tabla 2) y elasticidades (Tabla 3 ) se refieren a los mejores modelos de demanda por importaciones de manzanas frescas chilenas estimados, es decir, aquellos modelos con el mayor coeficiente de correlación múltiple, y donde para cada modelo se verificó el cumplimiento de los supuestos de no existencia de multicolinealidad, heterocedasticidad y autocorrelación.

Los modelos DDL estimados para Alemania sugieren que la demanda por importaciones de manzanas frescas chilenas depende del gasto en consumo per cápita, la variación del tipo de cambio real, y que las manzanas frescas chilenas compiten en ese mercado con las manzanas provenientes de Sudáfrica, como en (7), y Nueva Zelanda, como en (8).

$\ln _{\mathrm{AL}} \quad 69,921 \quad 5,461 \ln \mathrm{GCPC}_{\mathrm{AL}} \quad 1,525 \mathrm{VTCR}_{\mathrm{AL}} \quad 0,496 \ln \mathrm{PCIF}_{\text {Chile,SD }}$

$\ln \mathrm{M}_{\mathrm{AL}} \quad 74,697 \quad 5,9731 \ln \mathrm{GCPC}_{\mathrm{AL}} \quad 1.334 \mathrm{VTCR}_{\mathrm{AL}} \quad 0,566 \ln \mathrm{PCIF}_{\mathrm{Chile}, \mathrm{NZ}}$

Para Bélgica se estimó un modelo MDL, el cual sugiere que la demanda por importaciones de manzanas frescas chilenas depende del PIB per cápita a poder de paridad de compra, la variación del tipo de cambio real, y que las manzanas frescas chilenas compiten en ese mercado con las manzanas provenientes de Sudáfrica, como en (9).

$\mathrm{M}_{\mathrm{BE}}=1,9 \mathrm{E}-+167,157 \mathrm{PIBPPC}_{\mathrm{BE}}-8.769 .735 \mathrm{VTCR}_{\mathrm{BE}}-1,201 \mathrm{PCIF}_{\mathrm{Chile}, \mathrm{SD}}$
El modelo DDL estimado para España sugiere que la demanda por importaciones de manzanas frescas chilenas depende del gasto en consumo per cápita, que las manzanas frescas chilenas compiten en ese mercado con las manzanas provenientes de Nueva Zelanda, y que las importaciones fueron afectadas por el cambio puntual observado durante el periodo 1991 a 1992, como en (10).

$\ln \mathrm{M}_{\mathrm{ES}}=10,357+0,0007276 \mathrm{GCPC}_{\mathrm{ES}}-3.593 \mathrm{PCIF}_{\mathrm{Chile,NZ}}+0,914 \mathrm{D}$

Para Francia se estimó un modelo DSL, el cual sugiere que la demanda por importaciones de manzanas frescas chilenas depende del precio relativo de las manzanas provenientes de Argentina con respecto al precio de las manzanas de EE.UU., y del precio de las manzanas frescas chilenas con un rezago de dos periodos, como en (11).

$\ln \mathrm{M}_{\mathrm{FR}}=18,8491,951 \mathrm{PCIF}_{\mathrm{AR}, \mathrm{USA}} 3,654 \mathrm{PCIF}_{\text {Chile } 2}$

En el caso de Holanda, el modelo MDL estimado sugiere que la demanda por importaciones de manzanas frescas chilenas depende del precio relativo de las manzanas frescas chilenas con respecto al precio de las manzanas provenientes de Nueva Zelanda, del precio relativo de las manzanas frescas chilenas con un rezago de dos periodos, de la variación del tipo de cambio real, y de las importaciones que fueron afectadas por el cambio puntual observado durante el periodo 1991 a 1992, como en (12).

$\ln \mathrm{M}_{\mathrm{HO}}=16,663-0,708 \operatorname{lnPCIF} \mathrm{Chile} \mathrm{NZ}_{\mathrm{N}}+1,006 \operatorname{lnPCIF}_{\mathrm{CH}-2}-0,538 \mathrm{VTCR}_{\mathrm{HO}}=0,394 \mathrm{D}$

TABLA 1 - Estadísticas de las variables utilizadas en las ecuaciones de demanda por importaciones de manzanas chilenas

\begin{tabular}{|c|c|c|c|c|c|c|}
\hline Variable & & Alemania & Bélgica & España & Francia & Holanda \\
\hline \multirow{2}{*}{$\mathrm{M}^{\mathrm{a}}$} & Media & 14.179 .225 & 7.631 .358 & 18.689 .260 & 6.615 .614 & 72.445 .436 \\
\hline & D.S. & 8.964 .616 & 5.269 .689 & 14.077 .662 & 3.658 .140 & 19.081 .996 \\
\hline \multirow[t]{2}{*}{$\mathrm{PIBPC}^{\mathrm{b}}$} & Media & 32.593 & 30.856 & 18.387 & 30.119 & 32.530 \\
\hline & D.S. & 3.451 & 3.534 & 2.804 & 2.976 & 4.165 \\
\hline \multirow{2}{*}{$\mathrm{GCPC}^{\mathrm{b}}$} & Media & 18.584 & 16.689 & 11.052 & 16.813 & 16.350 \\
\hline & D.S. & 1.910 & 1.800 & 1.598 & 1.366 & 1.878 \\
\hline \multirow{2}{*}{ PIBPPC $^{c}$} & Media & 24.376 & 24.556 & 17.930 & 24.052 & 24.666 \\
\hline & D.S. & 2.665 & 2.628 & 2.822 & 2.297 & 3.349 \\
\hline \multirow{2}{*}{ IPC } & Media & 94,79 & 96,03 & 90,85 & 95,14 & 98,04 \\
\hline & D.S. & 11,84 & 12,07 & 22,60 & 11,82 & 13,03 \\
\hline \multirow{2}{*}{$\mathrm{HICPs}_{\mathrm{s}}$} & Media & 101,83 & 101,83 & 101,83 & 101,83 & 101,83 \\
\hline & D.S. & 7,33 & 7,33 & 7,33 & 7,33 & 7,33 \\
\hline \multirow{2}{*}{ IPIE } & Media & 95,94 & 95,94 & 95,94 & 95,94 & 95,94 \\
\hline & D.S. & 6,35 & 6,35 & 6,35 & 6,35 & 6,35 \\
\hline \multirow{2}{*}{$\mathrm{TCR}^{\mathrm{d}}$} & Media & 1,62 & 27,83 & 83,28 & 4,69 & 1,85 \\
\hline & D.S. & 0,45 & 17,23 & 52,84 & 2,23 & 0,58 \\
\hline \multirow{2}{*}{ VTCR } & Media & $-0,02$ & $-0,04$ & 0,00 & $-0,03$ & $-0,02$ \\
\hline & D.S. & 0,17 & 0,26 & 0,27 & 0,23 & 0,18 \\
\hline \multirow{2}{*}{ PCIF $_{\text {Chile }}{ }^{e}$} & Media & 0,46 & 0,46 & 0,55 & 0,55 & 0,49 \\
\hline & D.S. & 0,10 & 0,11 & 0,27 & 0,12 & 0,11 \\
\hline \multirow{2}{*}{$\mathrm{PCIF}_{\text {Chile,AR }}{ }^{\mathrm{e}}$} & Media & 1,01 & 1,02 & 1,25 & 1,20 & 1,11 \\
\hline & D.S. & 0,35 & 0,32 & 0,77 & 0,33 & 0,40 \\
\hline \multirow{2}{*}{$\mathrm{PCIF}_{\mathrm{Chile}, \mathrm{AU}}{ }^{\mathrm{e}}$} & Media & 0,67 & 0,66 & 0,76 & 0,79 & 0,71 \\
\hline & D.S. & 0,18 & 0,17 & 0,39 & 0,22 & 0,19 \\
\hline \multirow{2}{*}{$\mathrm{PCIF}_{\text {Chile,BR }}{ }^{\mathrm{e}}$} & Media & 0,94 & 0,93 & 1,18 & 1,12 & 1,02 \\
\hline & D.S. & 0,30 & 0,28 & 0,58 & 0,31 & 0,32 \\
\hline \multirow{2}{*}{$\mathrm{PCIF}_{\text {Chile,NZ }}{ }^{\mathrm{e}}$} & Media & 0,69 & 0,68 & 0,77 & 0,81 & 0,74 \\
\hline & D.S. & 0,24 & 0,22 & 0,44 & 0,27 & 0,24 \\
\hline \multirow{2}{*}{$\mathrm{PCIF}_{\text {Chile,SD }}{ }^{\mathrm{e}}$} & Media & 1,12 & 1,10 & 1,24 & 1,31 & 1,19 \\
\hline & D.S. & 0,37 & 0,36 & 0,64 & 0,44 & 0,40 \\
\hline
\end{tabular}

Fuente: Datos de la investigación.

a Cantidades totales importadas, medida en kilogramos netos.

${ }^{\mathrm{b}}$ Variable proxy del ingreso, medida en dólares de diciembre de 2003, deflactada por el IPC de cada país.

${ }^{\mathrm{c}}$ Variable proxy del ingreso, medida en dólares de diciembre de 2003, deflactada por el HICPs de la Unión Europea.

${ }^{\mathrm{d}}$ Tipo de cambio real, en términos de unidades de moneda local de cada país po r dólar.

${ }^{e}$ Precios CIF reales de importación, en dólares de diciembre de 2003, deflactados por IPIE de EE.UU. Las notaciones son AR: Argentina, AU: Australia, BR:

Brasil, NZ: Nueva Zelanda y SD: Sudáfrica. 
TABLA 2 - Coeficientes de los modelos de demanda por importaciones de manzanas chilenas

\begin{tabular}{|c|c|c|c|c|c|c|}
\hline Variable & Alemania $^{a}$ & Alemania $^{a}$ & Bélgica & España & Francia & Holanda \\
\hline Constante & $69,921(9.496)$ & $74,697(9,66)$ & $1,9 \mathrm{E}+07 \quad(7,378)$ & $10,357(21,035)$ & $18,849(21,519)$ & $16,663(38,48)$ \\
\hline $\ln \mathrm{GCPC}$ & $-5,461(-7,296)$ & $-5,973(-7,567)$ & & & & \\
\hline $\mathrm{GCPC}$ & & & & $7,276 \mathrm{E}-04(16,578)$ & & \\
\hline PIBPPC & & & $167,157(2,115)$ & & & \\
\hline $\ln \mathrm{PCIF}_{\mathrm{Chile}, \mathrm{SD}}$ & $-0,496(-2,303)$ & & $-1,3 \mathrm{E}+07(-6,508)$ & & & \\
\hline $\ln \mathrm{PCIF}_{\text {Chile, NZ }}$ & & $-0,566(-2,233)$ & & & & $-0,708(-3,414)$ \\
\hline$P C I F_{\text {Chile }, N Z}$ & & & & $-3,593(-9,79)$ & & \\
\hline$P C I F_{A R, E E . U U}$. & & & & & $-1,951(-4,136)$ & \\
\hline $\mathrm{PCIF}_{\text {Chile } 2}$ & & & & & $-3,654(-2,035)$ & \\
\hline $\ln \mathrm{PCIF}_{\text {Chile } 2}$ & & & & & & $1,006(2,851)$ \\
\hline VTCR & $-1,525(-3,494)$ & $-1,334(-3,075)$ & $-8.769 .735(-2,141)$ & & & $-0,538(-2,398)$ \\
\hline $\mathrm{D}$ & & & & $0,914(5,433)$ & & $0,394(2,975)$ \\
\hline $\mathrm{D}-\mathrm{W}$ & 1,998 & 1,918 & 2,212 & 2,339 & 1,661 & 2,207 \\
\hline $\mathrm{F}$ & 27,558 & 27,558 & 14,508 & 112,537 & 10,479 & 8,776 \\
\hline $\mathrm{R}^{2}$ & 0,849 & 0,846 & 0,744 & 0,963 & 0,583 & 0,730 \\
\hline $\mathrm{R}^{2}$-ajustado & 0,819 & 0,816 & 0,692 & 0,954 & 0,527 & 0,647 \\
\hline
\end{tabular}

Fuente: Datos de la investigación.

Entre paréntesis se indican los valores calculados del estadístico $t$-student.

${ }^{\text {a }}$ Son los mejores dos modelos estimados para Alemania.

TABLA 3 - Elasticidades de demanda por importaciones de manzanas chilenas

\begin{tabular}{|c|c|c|c|c|c|c|}
\hline Vari able & Alemania $^{\text {a }}$ & Alemania $^{a}$ & Bélgica & España & Francia & Holanda \\
\hline GCPC & $-5,461$ & $-5,973$ & & 8,035 & & \\
\hline PIBPPC & & & 0,538 & & & \\
\hline $\mathrm{PCIF}_{\text {Chile,SD }}$ & $-0,496$ & & $-0,696$ & & & \\
\hline $\mathrm{PCIF}_{\text {Chile,NZ }}$ & & $-0,566$ & & $-2,377$ & & $-0,708$ \\
\hline$P C I F_{A R, E E . U U}$ & & & & & $-1,34$ & \\
\hline PCIF $_{\text {Chile } 2}$ & & & & & $-1,58$ & 1,006 \\
\hline VTCR & $-0,027$ & $-0,023$ & $-0,047$ & & & $-0,010$ \\
\hline
\end{tabular}

Fuente: Datos de la investigación.

${ }^{a}$ Son los mejores dos modelos estimados para Alemania.

Las elasticidades ingreso obtenidas para Alemania $(-5,461$ y 5,973 ) sugieren que en ese país las manzanas chilenas son un bien inferior, mientras que en España $(8,035)$ se podrían clasificar en la categoría de bien de lujo; en el caso de Bélgica $(0,538)$ la demanda por manzanas chilenas es inelástica al ingreso, por lo que corresponderían a un bien normal. En Francia y Holanda los cambios en el ingreso no estarían explicando las variaciones en la demanda por manzanas chilenas.

En todos los países considerados en esta investigación, los principales competidores de las manzanas chilenas son las manzanas provenientes de Sudáfrica y Nueva Zelanda, excepto en Francia donde las manzanas chilenas compiten con las de Argentina y EE.UU. En Alemania la demanda es inelástica al precio de las manzanas chilenas con respecto al precio de las manzanas provenientes de Sudáfrica $(0,496)$ y Nueva Zelanda $(-0,566)$, por lo que los cambios en los precios relativos mencionados no afectarían significativamente la cantidad demandada por manzanas chilenas en Alemania. Una conclusión similar se puede inferir para el resultado de la elasticidad precio obtenida para Bélgica (0,696 ), aunque en este mercado las manzanas chilenas compiten solamente con las manzanas provenientes de Sudáfrica. En España las manzanas chilenas compiten fuertemente con las manzanas provenientes de Nueva Zelanda, considerando que se obtuvo una demanda elástica con respecto al precio relativo (-2,377). La demanda por manzanas chilenas es elástica al precio de las manzanas provenientes de Argentina con respecto al precio de EU en Francia (-1,34), lo cual sugiere que los cambios en dicho precio relativo afectarían más que proporcionalmente la cantidad demandada de manzanas chilenas en ese mercado; además, la cantidad demandada de manzanas chilenas en Francia estaría siendo explicada por el precio de las mismas manzanas con un rezago de dos periodos, considerando que la demanda en Francia $(-1,58)$ es elástica al precio rezagado. En Holanda se obtuvo que la demanda es inelástica al precio de las manzanas chilenas con respecto al precio de las manzanas provenientes de Nueva Zelanda $(-0,708)$, por lo que los cambios en este precio relativo no afectaría significativamente la cantidad demandada por manzanas chilenas en Holanda; además, la cantidad demandada de manzanas chilenas en Holanda estaría siendo explicada por el precio de las mismas manzanas con un rezago de dos periodos, considerando que la demanda en Holanda $(-1,006)$ es levemente elástica al precio rezagado.

Por otra parte, los resultados sugieren que la demanda por manzanas chilenas es inelástica a las variaciones en el tipo de cambio real en Alemania, Bélgica y Holanda; lo anterior significa que las variaciones en el tipo de cambio real no afectan significativamente la demanda por manzanas chilenas en cada uno de dichos países, en términos porcentuales.

Los resultados obtenidos en esta investigación son consistentes con los reportados por la literatura, en términos de las variables que estarían explicando la demanda por manzanas chilenas. La variable gasto en consumo per cápita es relevante en Alemania y España, tal como lo sugiere Agbola (2003) y Stewart et al. (2003), 
mientras que el ingreso per cápita es significativo en Bélgica (Cerda et al., 2004; Duval et al., 2002; Richards, 2000; Lee, 1994). El precio relativo es relevante en todos los modelos estimados, mientras que la variación del tipo de cambio real es significativa en Alemania, Bélgica y Holanda, tal como lo reportan Cerda et al. (2004) y Lee \& Fairchild (1988).

La clasificación de bien inferior para las manzanas frescas chilenas en Alemania podría explicarse porque durante todo el periodo de estudio se observó una tendencia decreciente en las importaciones desde ese país, lo cual pudo haber estado influenciado por variables no incluidas en esta investigación, tales como el mejor posicionamiento de otros productores en ese mercado a través de actividades de difusión y publicidad. El argumento inverso podría utilizarse para explicar la clasificación de bien de lujo de las manzanas frescas chilenas en España, considerando además que en este mercado la demanda es elástica al precio relativo de Chile con respecto al de Nueva Zelanda.

La demanda por manzanas frescas chilenas es inelástica al precio relativo en Alemania, Bélgica y Holanda en un rango -0,496 y -0,708. Estos valores son consistentes con los obtenidos por Cerda et al. (2004), Lee (1994), Arbola (2003), Lee et al. (1990), y Lee \& Fairchild (1988).

Finalmente, la demanda por manzanas frescas chilenas e s i nelástica a la variación del tipo de cambio real en Alemania, Bélgica y Holanda en un rango entre $-0,01 \mathrm{y}-0,047$, siendo consistente con las estimaciones de Cerda et al. (2004) y Lee \& Fairchild (1988), aún cuando la demanda responde de distinta forma a las variaciones del tipo de cambio real en cada mercado.

\section{CONCLUSIÓN}

En esta investigación se demuestra que:

1. La demanda por importaciones de manzanas frescas chilenas depende de las tradicionales variables ingreso o gasto en consumo per cápita,

2. Los precios relativos y la variación del tipo de cambio real juegan un papel destacado en la explicación de la demanda por importaciones y

3. La demanda por manzanas frescas exportadas por Chile a los países de la Unión Europea responde de manera diferente frente a los cambios en las variables explicativas en cada mercado. Esto último significa que frente a un cambio porcentual, igual y simultáneo, en alguna de las variables explicativas (ingreso o gasto, precio relativo, tipo de cambio) de cada país, el cambio porcentual de la demanda por importaciones de manzanas frescas chilenas será diferente en cada uno de los mercados analizados.

\section{REFERENCIAS}

AGBOLA, F.W. Estimation of food demand patterns in South Africa based on a survey of households. Journal of Agricultural and Applied Economics, v.35, n3, p.663-670, 2003.

BANCO MUNDIAL. 2004. Base de Datos (en línea). Disponible en: http:/ /www.bancomundial.org. Leído el 3 de mayo de 2004.
BLS. 2004. U.S. Import and Export Price Indexes (IEPI). Bureau of Labour Statistics. Available at: http://www.bls.gov/mxp/home.htm\#tables. Accessed 8 Jun, 2004

CERDA, A., G. LOBOS, E. KUFFERATH, \& SÁNCHEZ, F. Elasticidades de demanda por manzanas chilenas en el mercado de la Unión Europea: una estimación econométrica. Agricultura Técnica (Chile), v.64, n.4, p.399-408, 2004.

DEATON, A., \& MUELLBAUER, J. An Almost Ideal Demand System. The American Economic Review, v.70, n.3, p.312-325, 1980.

DIXON, R. Hybrid corn revisited. Econometrica, v.48, p.1451-1461, 1980.

DUVAL, Y., \& BIERE, A. Product diffusion and demand for new food products. Agribusiness, v.18, n1, p.23-36, 2002.

ECB. 2004. Harmonised Indices of Consumer Prices (HICPs). European Central Bank. Available at: http://www.ecb.int. Accessed 5 May, 2004.

EUROSTAT. 2004. Base de Datos (en línea). Disponible en: http:// www.eurostat.org. Leído el 10 de mayo de 2004.

FAOSTAT. 2004. Base de Datos (en línea). Disponible en: http:// www.faostat.fao.org. Leído el 24 de mayo de 2004.

FAS/USDA. 2004. United States Department of Agriculture, Foreign Agricultural Services (FAS online). Disponible en: http:// www.fas.usda.gov/. Leído el 14 de Junio de 2004.

GUJARATI, D. Econometría. México: McGraw Hill. $3^{a}$ ed., 2000. 824 p.

KAISER, H.M., D.J. LIU \& CONSIGNADO, T. An economic analysis of California raisin export promotion. Agribusiness, v.19, n.2, p.189201,2003.

LEE, J., \& FAIRCHILD, G.F. Exchange rates and foreign demand for US fresh grapefruit. Agribusiness, v.4, n.3, p.261-270, 1988.

LEE, J-Y. Fresh fruit consumption in Japan. Agribusiness, v.10, n.6, p.513$520,1994$.

LEE, J-Y., J.L. SEALE, \& JIERWIRIYAPANT, P.A. Do trade agreements help US exports? A study of the Japanese citrus industry. Agribusiness, v.6, n.5, p.505-514, 1990.

ODEPA. 2004. Base de datos de comercio exterior. Oficina de Estudios y Políticas Agrarias. Disponible en: http://www.odepa.cl. Leído el 29 de abril de 2004.

OSSA, F. 2001. La teoría de la Paridad del Poder de Compra de las monedas y el tipo de cambio flexible. Pontificia Universidad Católica de Chile, Instituto de Economía. Santiago, Chile. Documento de Trabajo, $\mathrm{N}^{\circ}$ 194 (abril), 33 p. Disponible en: http://www.economia.puc.cl/ publicaciones/Doc.Trabajo/Dt_194.pdf. Leído el 15 abril de 2004.

RICHARDS, T.J. A discrete/continuous model of fruit promotion, advertising, and response segmentation. Agribusiness, v.16, n.2, p.79-196, 2000.

STEWART, H., N. BLISARD, \& JOLLIFE, D. Do income constraints inhibit spending on fruits and vegetables among low-income households? Journal of Agricultural and Resource Economics, v.28, n.3, p.465480, 2003.

ZELLNER, A. An efficient method of estimating Seemingly Unrelated Regressions and test of aggregation bias. Journal of the American Statistical Association, v.57, p.348-368, 1962. 